\title{
Double Sequence Spaces by Means of Orlicz Functions
}

\author{
Abdullah Alotaibi, ${ }^{1}$ M. Mursaleen, ${ }^{2}$ and Kuldip Raj $^{3}$ \\ ${ }^{1}$ Department of Mathematics, Faculty of Science, King Abdulaziz University, P.O. Box 80203, Jeddah 21589, Saudi Arabia \\ ${ }^{2}$ Department of Mathematics, Aligarh Muslim University, Aligarh 202002, India \\ ${ }^{3}$ School of Mathematics, Shri Mata Vaishno Devi University, Katra, Jammu and Kashmir 182320, India
}

Correspondence should be addressed to M. Mursaleen; mursaleenm@gmail.com

Received 8 April 2014; Accepted 23 July 2014; Published 18 August 2014

Academic Editor: Ljubisa Kocinac

Copyright (c) 2014 Abdullah Alotaibi et al. This is an open access article distributed under the Creative Commons Attribution License, which permits unrestricted use, distribution, and reproduction in any medium, provided the original work is properly cited.

We define some classes of double entire and analytic sequences by means of Orlicz functions. We study some relevant algebraic and topological properties. Further some inclusion relations among the classes are also examined.

\section{Introduction and Preliminaries}

Of the definitions of convergence commonly employed for double series, only that due to Pringsheim permits a series to converge conditionally. Therefore, in spite of any disadvantages which it may possess, this definition is better adapted than others to the study of many problems in double sequences and series. Chief among the reasons why the theory of double sequences, under the Pringsheim definition of convergence, present difficulties not encountered in the theory of simple sequences, is the fact that a double sequence $\left(x_{i j}\right)$ may converge without $x_{i j}$ being a bounded function of $i$ and $j$. Thus it is not surprising that many authors in dealing with the convergence of double sequences should have restricted themselves to the class of bounded sequences or in dealing with the summability of double series to the class of series for which the function whose limit is the sum of the series is a bounded function of $i$ and $j$. Without such a restriction, peculiar things may sometimes happen; for example, a double power series may converge with partial sum $\left(S_{i j}\right)$ unbounded at a place exterior to its associated circles of convergence. Nevertheless there are problems in the theory of double sequences and series where this restriction of boundedness as it has been applied is considerably more stringent than need be. The initial works on double sequences are found in Bromwich [1]. Later on, it was studied by Hardy [2], Móricz [3], Móricz and Rhoades [4], Basarir and Sonalcan [5], and many others. Hardy [2] introduced the notion of regular convergence for double sequences. Mursaleen and Mohiuddine $[6,7]$ have characterized four dimensional matrix transformations between double sequences $x=\left(x_{m, n}\right)$. A good account of the study of double sequences can be found in most recent monograph by Mursaleen and Mohiuddine [8]. More recently, Altay and Başar [9] have defined the spaces $\mathscr{B} \mathcal{S}, \mathscr{B} \mathcal{S}(t), \mathscr{C} \mathcal{S}_{p}, \mathscr{C} \mathcal{S}_{b p}$, $\mathscr{C} \mathcal{S}_{r}$, and $\mathscr{B} \mathscr{V}$ of double sequences consisting of all double series whose sequence of partial sums is in the spaces $\mathscr{M}_{u}$, $\mathscr{M}_{u}(t), \mathscr{C}_{p}, \mathscr{C}_{b p}, \mathscr{C}_{r}$, and $\mathscr{L}_{u}$, respectively, and also examined some properties of these sequence spaces and determined the $\alpha$-duals of the spaces $\mathscr{B} \mathcal{S}, \mathscr{B} \mathscr{V}$, and $\mathscr{C} \mathcal{S}_{b p}$ and the $\beta(v)$-duals of the spaces $\mathscr{C} \mathcal{S}_{b p}$ and $\mathscr{C} \mathcal{S}_{r}$ of double series. Now, recently, Başar and Sever [10] have introduced the Banach space $\mathscr{L}_{q}$ of double sequences corresponding to the well-known space $\ell_{q}$ of single sequences and examined some properties of the space $\mathscr{L}_{q}$. By the convergence of a double sequence we mean the convergence in the Pringsheim sense; that is, a double sequence $x=\left(x_{k, l}\right)$ has Pringsheim limit $L$ (denoted by $P-\lim x=L)$ provided that given $\epsilon>0$ there exists $n \in N$ such that $\left|x_{k, l}-L\right|<\epsilon$ whenever $k, l>n$; see [11]. We will write more briefly as $P$-convergent. The double sequence $x=\left(x_{k, l}\right)$ is bounded if there exists a positive number $M$ such that $\left|x_{k, l}\right|<M$ for all $k$ and $l$.

An Orlicz function $M:[0, \infty) \rightarrow[0, \infty)$ is a continuous, nondecreasing, and convex function such that $M(0)=$ $0, M(x)>0$ for $x>0$ and $M(x) \rightarrow \infty$ as $x \rightarrow$ 
$\infty$. Lindenstrauss and Tzafriri [12] used the idea of Orlicz function to define the following sequence space. Let $w$ be the space of all real or complex sequences $x=\left(x_{k}\right)$; then

$$
l_{M}=\left\{x \in w: \sum_{k=1}^{\infty} M\left(\frac{\left|x_{k}\right|}{\rho}\right)<\infty, \text { for some } \rho>0\right\}
$$

which is called as an Orlicz sequence space. Also $l_{M}$ is a Banach space with the norm

$$
\|x\|=\inf \left\{\rho>0: \sum_{k=1}^{\infty} M\left(\frac{\left|x_{k}\right|}{\rho}\right) \leq 1\right\} .
$$

Also, it was shown in [13] that every Orlicz sequence space $l_{M}$ contains a subspace isomorphic to $l_{p}(p \geq 1)$. The $\Delta_{2}-$ condition is equivalent to $M(L x) \leq L M(x)$, for all $L$ with $0<L<1$. An Orlicz function $M$ can always be represented in the following integral form:

$$
M(x)=\int_{0}^{x} \eta(t) d t
$$

where $\eta$ is known as the kernel of $M$ and is right differentiable for $t \geq 0, \eta(0)=0$, and $\eta(t)>0 ; \eta$ is nondecreasing and $\eta(t) \rightarrow \infty$ as $t \rightarrow \infty$.

Let $X$ be a linear metric space. A function $p: X \rightarrow \mathbb{R}$ is called paranorm, if

(1) $p(x) \geq 0$, for all $x \in X$,

(2) $p(-x)=p(x)$, for all $x \in X$,

(3) $p(x+y) \leq p(x)+p(y)$, for all $x, y \in X$,

(4) if $\left(\lambda_{n}\right)$ is a sequence of scalars with $\lambda_{n} \rightarrow \lambda$ as $n \rightarrow$ $\infty$ and $\left(x_{n}\right)$ is a sequence of vectors with $p\left(x_{n}-x\right) \rightarrow$ 0 as $n \rightarrow \infty$, then $p\left(\lambda_{n} x_{n}-\lambda x\right) \rightarrow 0$ as $n \rightarrow$ $\infty$.

A paranorm $p$ for which $p(x)=0$ implies $x=0$ is called total paranorm and the pair $(X, p)$ is called a total paranormed space. It is well known that the metric of any linear metric space is given by some total paranorm (see [14], Theorem 10.4.2, p.183). For more details about sequence spaces see [13, 15-21].

A complex sequence, whose $k$ th term is $x_{k}$, is denoted by $\left\{x_{k}\right\}$. Let $\varphi$ be the set of all finite sequences. A sequence $x=$ $\left\{x_{k}\right\}$ is said to be analytic if $\sup _{k}\left|x_{k}\right|^{1 / k}<\infty$. The vector space of all analytic sequences will be denoted by $\Lambda$. A sequence $x$ is called entire sequence if $\lim _{k \rightarrow \infty}\left|x_{k}\right|^{1 / k}=0$. The vector space of all entire sequences will be denoted by $\Gamma$.

The notion of difference sequence spaces was introduced by Kizmaz [22], who studied the difference sequence spaces $l_{\infty}(\Delta), c(\Delta)$, and $c_{o}(\Delta)$. The notion was further generalized by Et and Çolak [23] by introducing the spaces $l_{\infty}\left(\Delta^{n}\right), c\left(\Delta^{n}\right)$, and $c_{o}\left(\Delta^{n}\right)$.

Let $r, s$ be nonnegative integers; then for $Z=l_{\infty}, c, c_{o}$ we have sequence spaces

$$
Z\left(\Delta_{s}^{r}\right)=\left\{x=\left(x_{k}\right) \in w:\left(\Delta_{s}^{r} x_{k}\right) \in Z\right\},
$$

where $\Delta_{s}^{r} x=\left(\Delta_{s}^{r} x_{k}\right)=\left(\Delta_{s}^{r-1} x_{k}-\Delta_{s}^{r-1} x_{k+1}\right)$ and $\Delta_{s}^{0} x_{k}=x_{k}$ for all $k \in \mathbb{N}$, which is equivalent to the following binomial representation:

$$
\Delta_{s}^{r} x_{k}=\sum_{v=0}^{r}(-1)^{v}\left(\begin{array}{l}
r \\
v
\end{array}\right) x_{k+s v}
$$

Taking $s=1$, we get the spaces which were studied by Et and Çolak [23]. Taking $r=s=1$, we get the spaces which were introduced and studied by Kizmaz [22].

Let $w^{\prime \prime}$ denote the space of all complex double sequences $x=\left(x_{k, l}\right)$. The space consisting of all those sequences $x$ in $w^{\prime \prime}$ such that $M_{k, l}\left(\left|x_{k, l}\right|^{1 / k+l} / \rho\right) \rightarrow 0$ as $k, l \rightarrow \infty$ for some arbitrary fixed $\rho>0$ is denoted by $\Gamma_{\mathscr{M}}^{2}$ and is known as double Orlicz space of entire sequences. The space $\Gamma_{\mathscr{M}}^{2}$ is a metric space with the metric $d(x, y)=\sup _{k, l} M_{k, l}\left(\left|x_{k, l}-y_{k, l}\right|^{1 / k+l} / \rho\right)$ for all $x=\left\{x_{k, l}\right\}$ and $y=\left\{y_{k, l}\right\}$ in $\Gamma_{\mathscr{M}}^{2}$.

The space consisting of all those sequences $x$ in $w^{\prime \prime}$ such that $\left.\sup _{k, l}\left(M_{k, l}\left(\left|x_{k, l}\right|^{1 / k+l} / \rho\right)\right)\right)<\infty$ for some arbitrarily fixed $\rho>0$ is denoted by $\Lambda_{\mathscr{M}}^{2}$ and is known as double Orlicz space of analytic sequences.

A double sequence space $E$ is said to be solid or normal if $\left(\alpha_{k, l} x_{k, l}\right) \in E$ whenever $\left(x_{k, l}\right) \in E$ and for all sequences of scalars $\left(\alpha_{k, l}\right)$ with $\left|\alpha_{k, l}\right| \leq 1$ (see [18]).

The following inequality will be used throughout the paper. Let $p=\left(p_{k, l}\right)$ be a double sequence of positive real numbers with $0<p_{k, l} \leq \sup _{k, l}=H$ and let $K=$ $\max \left\{1,2^{H-1}\right\}$. Then for the factorable sequences $\left\{a_{k, l}\right\}$ and $\left\{b_{k, l}\right\}$ in the complex plane, we have

$$
\left|a_{k, l}+b_{k, l}\right|^{p_{k, l}} \leq K\left(\left|a_{k, l}\right|^{p_{k, l}}+\left|b_{k, l}\right|^{p_{k, l}}\right)
$$

Let $\mathscr{M}=\left(M_{k, l}\right)$ be a sequence of Orlicz functions, let $p=$ $\left(p_{k, l}\right)$ be a bounded sequence of positive real numbers, let $u=$ $\left(u_{k, l}\right)$ be a sequence of strictly positive real numbers, and let $X$ be locally convex Hausdorff topological linear space whose topology is determined by a set of continuous seminorms $q$. The symbols $\Lambda^{2}(X), \Gamma^{2}(X)$ denote the space of all double analytic and double entire sequences, respectively, defined over $X$. In this paper we define the following sequence spaces:

$$
\begin{aligned}
\Lambda_{M}^{2}\left(\Delta_{s}^{r}, u, p, q\right) \\
=\left\{x \in \Lambda^{2}(X): \sup _{m, n} \frac{1}{m n}\right. \\
\quad \times \sum_{k, l=1,1}^{m, n} u_{k, l}\left[M_{k, l}\left(q\left(\frac{\left|\Delta_{s}^{r} x_{k, l}\right|^{1 / k+l}}{\rho}\right)\right)\right]^{p_{k, l}} \\
\quad<\infty, \text { for some } \rho>0\},
\end{aligned}
$$




$$
\begin{aligned}
\Gamma_{M}^{2}\left(\Delta_{s}^{r}, u, p, q\right) \\
=\left\{x \in \Gamma^{2}(X): \frac{1}{m n}\right. \\
\quad \times \sum_{k, l=1,1}^{m, n} u_{k, l}\left[M_{k, l}\left(q\left(\frac{\left|\Delta_{s}^{r} x_{k, l}\right|^{1 / k+l}}{\rho}\right)\right)\right]^{p_{k, l}} \\
\quad \longrightarrow 0 \text { as } m, n \rightarrow \infty, \text { for some } \rho>0\}
\end{aligned}
$$

The main aim of this paper is to introduce some double entire sequence spaces $\Lambda_{\mathscr{M}}^{2}\left(\Delta_{s}^{r}, u, p, q\right)$ and $\Gamma_{\mathscr{M}}^{2}\left(\Delta_{s}^{r}, u, p, q\right)$ defined by a sequence of Orlicz functions and study some topological properties and inclusion relation between these spaces.

\section{Main Results}

Theorem 1. Let $\mathscr{M}=\left(M_{k, l}\right)$ be a sequence of Orlicz functions, let $p=\left(p_{k, l}\right)$ be a bounded sequence of positive real numbers, and let $u=\left(u_{k, l}\right)$ be a sequence of strictly positive real numbers; then the spaces $\Gamma_{\mathscr{M}}^{2}\left(\Delta_{s}^{r}, u, p, q\right)$ and $\Lambda_{\mathscr{M}}^{2}\left(\Delta_{s}^{r}, u, p, q\right)$ are linear spaces over the field of complex numbers $\mathbb{C}$.

Proof. Let $x, y \in \Gamma_{\mathscr{M}}^{2}\left(\Delta_{s}^{r}, u, p, q\right)$ and $\alpha, \beta \in \mathbb{C}$. In order to prove the result, we need to find some $\rho_{3}>0$ such that

$$
\begin{aligned}
& \frac{1}{m n} \sum_{k, l=1,1}^{m, n} u_{k, l}\left[M_{k, l}\left(q\left(\frac{\left|\Delta_{s}^{r}\left(\alpha x_{k, l}+\beta y_{k, l}\right)\right|^{1 /(k+l)}}{\rho_{3}}\right)\right)\right]^{p_{k, l}} \\
& \quad \longrightarrow 0 \text { as } m, n \longrightarrow \infty .
\end{aligned}
$$

Since $x, y \in \Gamma_{\mathscr{M}}^{2}\left(\Delta_{s}^{r}, u, p, q\right)$, there exist some positive $\rho_{1}$ and $\rho_{2}$ such that

$$
\begin{aligned}
& \frac{1}{m n} \sum_{k, l=1,1}^{m, n} u_{k, l}\left[M_{k, l}\left(q\left(\frac{\left|\Delta_{s}^{r} x_{k, l}\right|^{1 /(k+l)}}{\rho_{1}}\right)\right)\right]^{p_{k, l}} \\
& \longrightarrow 0 \text { as } m, n \longrightarrow \infty, \\
& \frac{1}{m n} \sum_{k, l=1,1}^{m, n} u_{k, l}\left[M_{k, l}\left(q\left(\frac{\left|\Delta_{s}^{r} y_{k, l}\right|^{1 /(k+l)}}{\rho_{2}}\right)\right)\right]^{p_{k, l}} \\
& \longrightarrow 0 \text { as } m, n \longrightarrow \infty .
\end{aligned}
$$

Since $\mathscr{M}=\left(M_{k, l}\right)$ is a nondecreasing convex function, $q$ is a seminorm and $\Delta_{s}^{r}$ is linear and so, by using inequality (6), we have

$$
\begin{aligned}
& \frac{1}{m n} \sum_{k, l=1,1}^{m, n} u_{k, l}\left[M_{k, l}\left(q\left(\frac{\left|\Delta_{s}^{r}\left(\alpha x_{k, l}+\beta y_{k, l}\right)\right|^{1 /(k+l)}}{\rho_{3}}\right)\right)\right]^{p_{k, l}} \\
& \leq \frac{1}{(m n)} \\
& \quad \times \sum_{k, l=1,1}^{m, n} u_{k, l}\left[M_{k, l}\left(q\left(\frac{\alpha^{1 /(k+l)}\left|\Delta_{s}^{r} x_{k, l}\right|^{1 /(k+l)}}{\rho_{3}}\right)\right]^{\beta^{1 /(k+l)}\left|\Delta_{s}^{r} y_{k, l}\right|^{1 /(k+l)}}\right. \\
& \rho_{3}
\end{aligned}
$$

Take $\rho_{3}>0$ such that $1 / \rho_{3}=\min \left\{1 / \alpha \rho_{1}, 1 / \beta \rho_{2}\right\}$ :

$$
\begin{aligned}
& \frac{1}{m n} \sum_{k, l=1,1}^{m, n} u_{k, l}\left[M_{k, l}\left(q\left(\frac{\left|\Delta_{s}^{r}\left(\alpha x_{k, l}+\beta y_{k, l}\right)\right|^{1 /(k+l)}}{\rho_{3}}\right)\right)\right]^{p_{k, l}} \\
& \leq \frac{1}{m n} \sum_{k, l=1,1}^{m, n} u_{k, l}\left[M _ { k , l } \left(q \left(\frac{\left|\Delta_{s}^{r} x_{k, l}\right|^{1 /(k+l)}}{\rho_{1}}\right.\right.\right. \\
& \left.\left.\left.+\frac{\left|\Delta_{s}^{r} y_{k, l}\right|^{1 /(k+l)}}{\rho_{2}}\right)\right)\right]^{p_{k, l}} \\
& \leq \frac{1}{m n} \sum_{k, l=1,1}^{m, n} u_{k, l}\left[M_{k, l}\left(q\left(\frac{\left|\Delta_{s}^{r} x_{k, l}\right|^{1 /(k+l)}}{\rho_{1}}\right)\right)^{p_{k, l}}\right. \\
& \left.+u_{k, l} M_{k, l}\left(q\left(\frac{\left|\Delta_{s}^{r} y_{k, l}\right|^{1 /(k+l)}}{\rho_{2}}\right)\right)^{p_{k, l}}\right] \\
& \leq K \frac{1}{m n} \sum_{k, l=1,1}^{m, n} u_{k, l}\left[M_{k, l}\left(q\left(\frac{\left|\Delta_{s}^{r} x_{k, l}\right|^{1 /(k+l)}}{\rho_{1}}\right)\right)\right]^{p_{k, l}} \\
& +K \frac{1}{m n} \sum_{k, l=1,1}^{m, n} u_{k, l}\left[M_{k, l}\left(q\left(\frac{\left|\Delta_{s}^{r} y_{k, l}\right|^{1 /(k+l)}}{\rho_{2}}\right)\right)\right]^{p_{k, l}} \\
& \longrightarrow 0 \text { as } m, n \longrightarrow \infty \text {. }
\end{aligned}
$$

Hence

$$
\begin{aligned}
& \sum_{k, l=1,1}^{m, n} u_{k, l}\left[M_{k, l}\left(q\left(\frac{\left|\alpha \Delta_{s}^{r} x_{k, l}+\beta \Delta_{s}^{r} y_{k, l}\right|^{1 /(k+l)}}{\rho_{3}}\right)\right)\right]^{p_{k, l}} \longrightarrow 0 \\
& \text { as } m, n \rightarrow \infty \text {. }
\end{aligned}
$$

This proves that $\Gamma_{\mathscr{M}}^{2}\left(\Delta_{s}^{r}, u, p, q\right)$ is a linear space. Similarly, we can prove $\Lambda_{\mathscr{M}}^{2}\left(\Delta_{s}^{r}, u, p, q\right)$ is a linear space. 
Theorem 2. Let $\mathscr{M}=\left(M_{k, l}\right)$ be a sequence of Orlicz functions, let $p=\left(p_{k, l}\right)$ be a bounded sequence of positive real numbers, and let $u=\left(u_{k, l}\right)$ be a sequence of strictly positive real numbers; then the space $\Gamma_{\mathscr{M}}^{2}\left(\Delta_{s}^{r}, u, p, q\right)$ is a paranormed space with paranorm defined by

$$
\begin{aligned}
& g_{\Delta}(x)=\inf \left\{\rho^{\rho_{m, n} / H}:\right. \\
& \quad \sup _{k, l \geq 1} u_{k, l}\left[M_{k, l}\left(q\left(\frac{\left|\Delta_{s}^{r} x_{k, l}\right|^{1 /(k+l)}}{\rho}\right)\right)\right]^{p_{k, l}} \\
& \quad \leq 1 ; \rho>0\},
\end{aligned}
$$

where $H=\max \left(1, \sup _{k, l} p_{k, l}\right)$.

Proof. Clearly $g_{\Delta}(x) \geq 0, g_{\Delta}(x)=g_{\Delta}(-x)$ and $g_{\Delta}(\bar{\theta})=$ 0 , where $\theta$ is the zero sequence of $X$. For $\left(x_{k, l}\right),\left(y_{k, l}\right) \in$ $\Gamma_{\mathscr{M}}^{2}\left(\Delta_{s}^{r}, \mathcal{u}, p, q\right)$, there exist $\rho_{1}, \rho_{2}>0$ such that

$$
\begin{aligned}
& \sup _{k, l \geq 1} u_{k, l}\left[M_{k, l}\left(q\left(\frac{\left|\Delta_{s}^{r} x_{k, l}\right|^{1 /(k+l)}}{\rho_{1}}\right)\right)\right]^{p_{k, l}} \leq 1, \\
& \sup _{k, l \geq 1} u_{k, l}\left[M_{k, l}\left(q\left(\frac{\left|\Delta_{s}^{r} y_{k, l}\right|^{1 /(k+l)}}{\rho_{2}}\right)\right)\right]^{p_{k, l}} \leq 1 .
\end{aligned}
$$

Suppose that $\rho=\rho_{1}+\rho_{2}$; then

$$
\begin{aligned}
\sup _{k, l \geq 1} u_{k, l} & {\left[M_{k, l}\left(q\left(\frac{\left|\Delta_{s}^{r}\left(x_{k, l}+y_{k, l}\right)\right|^{1 /(k+l)}}{\rho}\right)\right)\right]^{p_{k, l}} } \\
\leq & \left(\frac{\rho_{1}}{\rho_{1}+\rho_{2}}\right) \sup _{k, l \geq 1} u_{k, l}\left[M_{k, l}\left(q\left(\frac{\left|\Delta_{s}^{r} x_{k, l}\right|^{1 /(k+l)}}{\rho_{1}}\right)\right)\right]^{p_{k, l}} \\
& +\left(\frac{\rho_{2}}{\rho_{1}+\rho_{2}}\right) \\
& \times \sup _{k, l \geq 1} u_{k, l}\left[M_{k, l}\left(q\left(\frac{\left|\Delta_{s}^{r} y_{k, l}\right|^{1 /(k+l)}}{\rho_{2}}\right)\right)\right]^{p_{k, l}} \leq 1 .
\end{aligned}
$$

Hence

$$
\begin{aligned}
& g_{\Delta}(x+y) \\
& \leq \inf \left\{\left(\rho_{1}+\rho_{2}\right)^{p_{m, n} / H}:\right. \\
& \quad \sup _{k, l \geq 1} u_{k, l}\left[M_{k, l}\left(q\left(\frac{\left|\Delta_{s}^{r} x_{k, l}\right|^{1 /(k+l)}}{\rho_{1}+\rho_{2}}\right)\right)\right]^{p_{k, l}} \\
& \left.\leq 1, \rho_{1}, \rho_{2}>0, m, n \in \mathbb{N}\right\}
\end{aligned}
$$

$$
\begin{aligned}
& \leq \inf \left\{\left(\rho_{1}\right)^{p_{m, n} / H}:\right. \\
& \quad \sup _{k, l \geq 1} u_{k, l}\left[M_{k, l}\left(q\left(\frac{\left|\Delta_{s}^{r} x_{k, l}\right|^{1 /(k+l)}}{\rho_{1}}\right)\right)\right]^{p_{k, l}} \\
& \left.\leq 1, \rho_{1}>0, m, n \in \mathbb{N}\right\} \\
& +\inf \left\{\left(\rho_{2}\right)^{p_{m, n} / H}:\right. \\
& \quad \sup _{k, l \geq 1}\left[M_{k, l}\left(q\left(\frac{\left|\Delta_{s}^{r} y_{k, l}\right|^{1 /(k+l)}}{\rho_{2}}\right)\right)\right]^{p_{k, l}} \\
& \left.\leq 1, \rho_{2}>0, m, n \in \mathbb{N}\right\} .
\end{aligned}
$$

Thus we have $g_{\Delta}(x+y) \leq g_{\Delta}(x)+g_{\Delta}(y)$. Hence $g_{\Delta}$ satisfies the triangle inequality. Now,

$$
\begin{gathered}
g_{\Delta}(\lambda x)=\inf \left\{(\rho)^{p_{m, n} / H}:\right. \\
\sup _{k, l \geq 1} u_{k, l}\left[M_{k, l}\left(q\left(\frac{\left|\lambda \Delta_{s}^{r} x_{k, l}\right|^{1 /(k+l)}}{\rho}\right)\right)\right]^{p_{k, l}} \\
\leq 1, \rho>0, m, n \in \mathbb{N}\} \\
=\inf \left\{(r|\lambda|)^{p_{m, n} / H}:\right. \\
\sup _{k, l \geq 1} u_{k, l}\left[M_{k, l}\left(q\left(\frac{\left|\Delta_{s}^{r} x_{k, l}\right|^{1 /(k+l)}}{r}\right)\right)\right]^{p_{k, l}} \\
\leq 1, r>0, m, n \in \mathbb{N}\},
\end{gathered}
$$

where $r=\rho /|\lambda|$. Hence $\Gamma_{\mathscr{M}}^{2}\left(\Delta_{s}^{r}, u, p, q\right)$ is a paranormed space.

Theorem 3. If $\mathscr{M}^{\prime}=\left(M_{k, l}^{\prime}\right)$ and $\mathscr{M}^{\prime \prime}=\left(M_{k, l}^{\prime \prime}\right)$ are two sequences of Orlicz functions, then

$$
\Gamma_{\mathscr{M}^{\prime}}^{2}\left(\Delta_{s}^{r}, u, p, q\right) \cap \Gamma_{\mathscr{M}^{\prime \prime}}^{2}\left(\Delta_{s}^{r}, u, p, q\right) \subseteq \Gamma_{\mathscr{M}^{\prime}+\mathscr{M}^{\prime \prime}}^{2}\left(\Delta_{s}^{r}, u, p, q\right) .
$$


Proof. Let $x \in \Gamma_{\mathscr{M}^{\prime}}^{2}\left(\Delta_{s}^{r}, u, p, q\right) \cap \Gamma_{\mathscr{M}^{\prime \prime}}^{2}\left(\Delta_{s}^{r}, u, p, q\right)$. Then there exist $\rho_{1}$ and $\rho_{2}$ such that

$$
\begin{aligned}
& \frac{1}{m n} \sum_{k, l=1,1}^{m, n} u_{k, l}\left[M_{k, l}^{\prime}\left(q\left(\frac{\left|\Delta_{s}^{r} x_{k, l}\right|^{1 /(k+l)}}{\rho_{1}}\right)\right)\right]^{p_{k, l}} \\
& \longrightarrow 0 \text { as } m, n \longrightarrow \infty, \\
& \frac{1}{m n} \sum_{k, l=1,1}^{m, n} u_{k, l}\left[M_{k, l}^{\prime \prime}\left(q\left(\frac{\left|\Delta_{s}^{r} x_{k, l}\right|^{1 /(k+l)}}{\rho_{2}}\right)\right)\right]^{p_{k, l}} \\
& \quad \longrightarrow 0 \text { as } m, n \longrightarrow \infty .
\end{aligned}
$$

Let $\rho=\min \left(1 / \rho_{1}, 1 / \rho_{2}\right)$. Then we have

$$
\begin{aligned}
& \frac{1}{m n} \sum_{k, l=1,1}^{m, n} u_{k, l}\left[\left(M_{k, l}^{\prime}+M_{k, l}^{\prime \prime}\right)\left(q\left(\frac{\left|\Delta_{s}^{r} x_{k, l}\right|^{1 /(k+l)}}{\rho}\right)\right)\right]^{p_{k, l}} \\
& \leq K\left[\frac{1}{m n} \sum_{k, l=1,1}^{m, n} u_{k, l}\left[M_{k, l}^{\prime}\left(q\left(\frac{\left|\Delta_{s}^{r} x_{k, l}\right|^{1 /(k+l)}}{\rho_{1}}\right)\right)\right]^{p_{k, l}}\right] \\
& \quad+K\left[\frac{1}{m n} \sum_{k, l=1,1}^{m, n} u_{k, l}\left[M_{k, l}^{\prime \prime}\left(q\left(\frac{\left|\Delta_{s}^{r} x_{k, l}\right|^{1 /(k+l)}}{\rho_{2}}\right)\right)\right]^{p_{k, l}}\right],
\end{aligned}
$$

$\longrightarrow 0$ as $m, n \longrightarrow \infty$

by (20). Therefore, $x \in \Gamma_{\mathscr{M}^{\prime}+\mathscr{M}^{\prime \prime}}^{2}\left(\Delta_{s}^{r}, u, p, q\right)$.

Theorem 4. Let $r \geq 1$. Then we have the following inclusions:

(i) $\Gamma_{\mathscr{M}}^{2}\left(\Delta_{s}^{r-1}, u, p, q\right) \subseteq \Gamma_{\mathscr{M}}^{2}\left(\Delta_{s}^{r}, u, p, q\right)$,

(ii) $\Lambda_{\mathscr{M}}^{2}\left(\Delta_{s}^{r-1}, u, p, q\right) \subseteq \Lambda_{\mathscr{M}}^{2}\left(\Delta_{s}^{r}, u, p, q\right)$.

Proof. Let $x \in \Gamma_{\mathscr{M}}^{2}\left(\Delta_{s}^{r-1}, u, p, q\right)$. Then we have

$$
\frac{1}{m n} \sum_{k, l=1,1}^{m, n} u_{k, l}\left[M_{k, l}\left(q\left(\frac{\left|\Delta_{s}^{r-1} x_{k, l}\right|^{1 /(k+l)}}{\rho}\right)\right)\right]^{p_{k, l}} \longrightarrow 0
$$

as $m, n \longrightarrow \infty, \quad$ for some $\rho>0$.
Since $\mathscr{M}=\left(M_{k, l}\right)$ is nondecreasing convex function and $q$ is a seminorm, we have

$$
\begin{aligned}
& \frac{1}{m n} \sum_{k, l=1,1}^{m, n} u_{k, l}\left[M_{k, l}\left(q\left(\frac{\left|\Delta_{s}^{r} x_{k, l}\right|^{1 /(k+l)}}{\rho}\right)\right)\right]^{p_{k, l}} \\
& \leq \frac{1}{m n} \\
& \times \sum_{k, l=1,1}^{m, n} u_{k, l} \\
& \times\left[M_{k, l}\left(q\left(\frac{\left|\Delta_{s}^{r-1} x_{k, l}-\Delta_{s}^{r-1} x_{k+1, l+1}\right|^{1 /(k+l)}}{\rho}\right)\right)\right]^{p_{k, l}} \\
& \leq K\left\{\frac{1}{m n} \sum_{k, l=1,1}^{m, n} u_{k, l}\left[M_{k, l}\left(q\left(\frac{\left|\Delta_{s}^{r-1} x_{k, l}\right|^{1 /(k+l)}}{\rho}\right)\right)\right]^{p_{k, l}}\right. \\
& +\frac{1}{m n} \\
& \times \sum_{k, l=1,1}^{m, n} u_{k, l} \\
& \left.\times\left[M_{k, l}\left(q\left(\frac{\left|\Delta_{s}^{r-1} x_{k+1, l+1}\right|^{1 /(k+l)}}{\rho}\right)\right)\right]^{p_{k, l}}\right\} \\
& \longrightarrow 0 \text { as } m, n \rightarrow \infty \text {. }
\end{aligned}
$$

Therefore, $(1 / m n) \sum_{k, l=1,1}^{m, n} u_{k, l}\left[M_{k, l}\left(q\left(\left|\Delta_{s}^{r} x_{k, l}\right|^{1 /(k+l)} / \rho\right)\right)\right]^{p_{k, l}}$

$\rightarrow 0$ as $m, n \rightarrow \infty$. Hence, $x \in \Gamma_{\mathscr{M}}^{2}\left(\Delta_{s}^{r}, u, p, q\right)$. This completes the proof of (i). Similarly, we can prove (ii).

Theorem 5. Let $0 \leq p_{k, l} \leq t_{k, l}$ and let $\left\{t_{k, l} / p_{k, l}\right\}$ be bounded. Then $\Gamma_{\mathscr{M}}^{2}\left(\Delta_{s}^{r}, u, t, q\right) \subset \Gamma_{\mathscr{M}}^{2}\left(\Delta_{s}^{r}, u, p, q\right)$.

Proof. Let $x \in \Gamma_{\mathscr{M}}^{2}\left(\Delta_{s}^{r}, u, t, q\right)$. Then

$$
\frac{1}{m n} \sum_{k, l=1,1}^{m, n} u_{k, l}\left[M_{k, l}\left(q\left(\frac{\left|\Delta_{s}^{r} x_{k, l}\right|^{1 /(k+l)}}{\rho}\right)\right)\right]^{t_{k, l}} \longrightarrow 0
$$

as $m, n \longrightarrow \infty$.

Let $w_{k, l}=(1 / m n) \sum_{k, l=1,1}^{m, n} u_{k, l}\left[M_{k, l}\left(q\left(\left|\Delta_{s}^{r} x_{k, l}\right|^{1 /(k+l)} / \rho\right)\right)\right]^{q_{k, l}}$ and $\lambda_{k, l}=p_{k, l} / t_{k, l}$. Since $p_{k, l} \leq t_{k, l}$, we have $0 \leq \lambda_{k, l} \leq 1$. Take $0<\lambda<\lambda_{k, l}$. Define

$$
\begin{aligned}
& u_{k, l}= \begin{cases}w_{k, l} & \text { if } w_{k, l} \geq 1 \\
0 & \text { if } w_{k, l}<1,\end{cases} \\
& v_{k, l}= \begin{cases}0 & \text { if } w_{k, l} \geq 1 \\
w_{k, l} & \text { if } \quad w_{k, l}<1,\end{cases}
\end{aligned}
$$


where $w_{k, l}=u_{k, l}+v_{k, l}$ and $w_{k, l}^{\lambda_{k, l}}=u_{k, l}^{\lambda_{k, l}}+v_{k, l}^{\lambda_{k, l}}$. It follows that $u_{k, l}^{\lambda_{k, l}} \leq u_{k, l} \leq w_{k, l}$ and $v_{k, l}^{\lambda_{k, l}} \leq v_{k, l}^{\lambda}$. Since $w_{k, l}^{\lambda_{k}}=u_{k, l}^{\lambda_{k}}+v_{k, l}^{\lambda_{k}}$, then $w_{k, l}^{\lambda_{k, l}} \leq w_{k, l}+v_{k, l}^{\lambda}$. Thus,

$$
\begin{aligned}
& \frac{1}{m n} \sum_{k, l=1,1}^{m, n} u_{k, l}\left[M_{k, l}\left(q\left(\frac{\left|\Delta_{s}^{r} x_{k, l}\right|^{1 /(k+l)}}{\rho}\right)\right)^{t_{k, l}}\right]^{\lambda_{k, l}} \\
& \leq \frac{1}{m n} \sum_{k, l=1,1}^{m, n} u_{k, l}\left[M_{k, l}\left(q\left(\frac{\left|\Delta_{s}^{r} x_{k, l}\right|^{1 /(k+l)}}{\rho}\right)\right)\right]^{t_{k, l}} \Longrightarrow \frac{1}{m n} \\
& \quad \times \sum_{k, l=1,1}^{m, n} u_{k, l}\left[M_{k, l}\left(q\left(\frac{\left|\Delta_{s}^{r} x_{k, l}\right|^{1 /(k+l)}}{\rho}\right)\right)^{t_{k, l}}\right]^{p_{k, l} / t_{k, l}} \\
& \leq \frac{1}{m n} \sum_{k, l=1,1}^{m, n} u_{k, l}\left[M_{k, l}\left(q\left(\frac{\left|\Delta_{s}^{m, n} x_{k, l}\right|^{1 /(k+l)}}{\rho}\right)\right)\right]^{t_{k, l}} \Longrightarrow \frac{1}{m n} \\
& \quad \times \sum_{k, l=1,1}^{m, n} u_{k, l}\left[M_{k, l}\left(q\left(\frac{\left|\Delta_{s}^{r} x_{k, l}\right|^{1 /(k+l)}}{\rho}\right)\right)\right]^{p_{k, l}} \\
& \leq \frac{1}{m n} \sum_{k, l=1,1}^{m, n} u_{k, l}\left[M_{k, l}\left(q\left(\frac{\left|\Delta_{s}^{r} x_{k, l}\right|^{1 /(k+l)}}{\rho}\right)\right)\right]^{t_{k, l}} \\
& \longrightarrow 0 \quad \text { as } m, n \longrightarrow \infty(\text { by }(24)) .
\end{aligned}
$$

Therefore,

$$
\frac{1}{m n} \sum_{k, l=1,1}^{m, n} u_{k, l}\left[M_{k, l}\left(q\left(\frac{\left|\Delta_{s}^{r} x_{k, l}\right|^{1 /(k+l)}}{\rho}\right)\right)\right]^{p_{k, l}} \longrightarrow 0
$$

as $m, n \rightarrow \infty$

Hence $x \in \Gamma_{\mathscr{M}}^{2}\left(\Delta_{s}^{r}, u, p, q\right)$. From (24), we get $\Gamma_{\mathscr{M}}^{2}\left(\Delta_{s}^{r}, u, t, q\right) \subset$ $\Gamma_{\mathscr{M}}^{2}\left(\Delta_{s}^{r}, u, p, q\right)$.

Theorem 6. (i) Let $0<\inf p_{k, l} \leq p_{k, l} \leq 1$. Then $\Gamma_{M}^{2}\left(\Delta_{s}^{r}, u\right.$, $p, q) \subset \Gamma_{\mathscr{M}}^{2}\left(\Delta_{s}^{r}, u, q\right)$. (ii) Let $1 \leq p_{k, l} \leq \sup p_{k, l}<\infty$. Then $\Gamma_{\mathscr{M}}^{2}\left(\Delta_{s}^{r}, u, q\right) \subset \Gamma_{\mathscr{M}}^{2}\left(\Delta_{s}^{r}, u, p, q\right)$.

Proof. (i) Let $x \in \Gamma_{M}^{2}\left(\Delta_{s}^{r}, u, p, q\right)$. Then

$$
\frac{1}{m n} \sum_{k, l=1,1}^{m, n} u_{k, l}\left[M_{k, l}\left(q\left(\frac{\left|\Delta_{s}^{r} x_{k, l}\right|^{1 /(k+l)}}{\rho}\right)\right)\right]^{p_{k, l}} \rightarrow 0
$$

Since $0<\inf p_{k, l} \leq p_{k, l} \leq 1$,

$$
\begin{aligned}
& \frac{1}{m n} \sum_{k, l=1,1}^{m, n} u_{k, l}\left[M_{k, l}\left(q\left(\frac{\left|\Delta_{s}^{r} x_{k, l}\right|^{1 /(k+l)}}{\rho}\right)\right)\right] \\
& \quad \leq \frac{1}{m n} \sum_{k, l=1,1}^{m, n} u_{k, l}\left[M_{k, l}\left(q\left(\frac{\left|\Delta_{s}^{r} x_{k, l}\right|^{1 /(k+l)}}{\rho}\right)\right)\right]^{p_{k, l}} \\
& \quad \longrightarrow 0 \text { as } m, n \longrightarrow \infty .
\end{aligned}
$$

From (28) and (29) it follows that $x \in \Gamma_{\mathscr{M}}^{2}\left(\Delta_{s}^{r}, u, q\right)$. Thus $\Gamma_{\mathscr{M}}^{2}\left(\Delta_{s}^{r}, u, p, q\right) \subset \Gamma_{\mathscr{M}}^{2}\left(\Delta_{s}^{r}, u, q\right)$.

(ii) Let $p_{k, l} \geq 1$ for each $k, l$ and $\sup p_{k, l}<\infty$ and let $x \in \Gamma_{\mathscr{M}}^{2}\left(\Delta_{s}^{r}, u, q\right)$. Then

$$
\begin{aligned}
& \frac{1}{m n} \sum_{k, l=1,1}^{m, n} u_{k, l}\left[M_{k, l}\left(q\left(\frac{\left|\Delta_{s}^{r} x_{k, l}\right|^{1 /(k+l)}}{\rho}\right)\right)\right] \\
& \longrightarrow 0 \text { as } m, n \longrightarrow \infty .
\end{aligned}
$$

Since $1 \leq p_{k, l} \leq \sup p_{k, l}<\infty$, we have

$$
\begin{aligned}
& \frac{1}{m n} \sum_{k, l=1,1}^{m, n} u_{k, l}\left[M_{k, l}\left(q\left(\frac{\left|\Delta_{s}^{r} x_{k, l}\right|^{1 /(k+l)}}{\rho}\right)\right)\right]^{p_{k, l}} \\
& \leq \frac{1}{m n} \sum_{k, l=1,1}^{m, n} u_{k, l}\left[M_{k, l}\left(q\left(\frac{\left|\Delta_{s}^{r} x_{k, l}\right|^{1 /(k+l)}}{\rho}\right)\right)\right] \\
& \longrightarrow 0 \quad \text { as } m, n \longrightarrow \infty .
\end{aligned}
$$

This implies that $x \in \Gamma_{\mathscr{M}}^{2}\left(\Delta_{s}^{r}, u, p, q\right)$. Therefore, $\Gamma_{\mathscr{M}}^{2}\left(\Delta_{s}^{r}\right.$, $u, q) \subset \Gamma_{\mathscr{M}}^{2}\left(\Delta_{s}^{r}, u, p, q\right)$.

Theorem 7. Suppose $(1 / m n) \sum_{k, l=1,1}^{m, n} u_{k, l}\left[M_{k, l}\left(q\left(\left|\Delta_{s}^{r} x_{k, l}\right|^{1 /(k+l)} /\right.\right.\right.$ $\rho))]^{p_{k, l}} \leq\left|x_{k, l}\right|^{1 / k+l}$; then $\Gamma^{2} \subset \Gamma_{\mathscr{M}}^{2}\left(\Delta_{s}^{r}, u, p, q\right)$.

Proof. Let $x \in \Gamma^{2}$. Then we have

$$
\left|x_{k, l}\right|^{1 / k+l} \longrightarrow 0 \quad \text { as } k, l \longrightarrow \infty \text {. }
$$

$\operatorname{But}(1 / m n) \sum_{k, l=1,1}^{m, n} u_{k, l}\left[M_{k, l}\left(q\left(\left|\Delta_{s}^{r} x_{k, l}\right|^{1 /(k+l)} / \rho\right)\right)\right]^{p_{k, l}} \leq\left|x_{k, l}\right|^{1 / k+l}$, by our assumption, implies that

$$
\frac{1}{m n} \sum_{k, l=1,1}^{m, n} u_{k, l}\left[M_{k, l}\left(q\left(\frac{\left|\Delta_{s}^{r} x_{k, l}\right|^{1 /(k+l)}}{\rho}\right)\right)\right]^{p_{k, l}} \longrightarrow 0
$$

as $m, n \longrightarrow \infty$ by (32) .

Then $x \in \Gamma_{\mathscr{M}}^{2}\left(\Delta_{s}^{r}, u, p, q\right)$ and $\Gamma^{2} \subset \Gamma_{\mathscr{M}}^{2}\left(\Delta_{s}^{r}, u, p, q\right)$.

Theorem 8. $\Gamma_{\mathscr{M}}^{2}\left(\Delta_{s}^{r}, u, p, q\right)$ is solid.

Proof. Let $\left(x_{k, l}\right) \in \Gamma_{\mathscr{M}}^{2}\left(\Delta_{s}^{r}, u, p, q\right)$; then

$$
\frac{1}{m n} \sum_{k, l=1,1}^{m, n} u_{k, l}\left[M_{k, l}\left(q\left(\frac{\left|\Delta_{s}^{r} x_{k, l}\right|^{1 /(k+l)}}{\rho}\right)\right)\right]^{p_{k, l}} \longrightarrow 0
$$

$$
\text { as } m, n \longrightarrow \infty, \quad \text { for some } \rho>0 \text {. }
$$


Let $\left(\alpha_{k, l}\right)$ be a double sequence of scalars such that $\left|\alpha_{k, l}\right| \leq 1$ for all $k, l \in \mathbb{N} \times \mathbb{N}$. Then we have

$$
\begin{array}{r}
\frac{1}{m n} \sum_{k, l=1,1}^{m, n} u_{k, l}\left[M_{k, l}\left(q\left(\frac{\left|\Delta_{s}^{r} \alpha_{k, l} x_{k, l}\right|^{1 /(k+l)}}{\rho}\right)\right)\right]^{p_{k, l}} \\
\leq \frac{1}{m n} \sum_{k, l=1,1}^{m, n} u_{k, l}\left[M_{k, l}\left(q\left(\frac{\left|\Delta_{s}^{r} x_{k, l}\right|^{1 /(k+l)}}{\rho}\right)\right)\right]^{p_{k, l}} \longrightarrow 0 \\
\text { as } m, n \rightarrow \infty
\end{array}
$$

and this completes the proof.

Corollary 9. $\Gamma_{\mathscr{M}}^{2}\left(\Delta_{s}^{r}, u, p, q\right)$ is monotone.

Proof. It is obvious.

\section{Conflict of Interests}

The authors declare that there is no conflict of interests regarding the publication of this paper.

\section{Acknowledgment}

The authors gratefully acknowledge the financial support from King Abdulaziz University, Jeddah, Saudi Arabia.

\section{References}

[1] T. J. Bromwich, An Introduction to the Theory of Infinite Series, Macmillan, New York, NY, USA, 1965.

[2] G. H. Hardy, "On the convergence of certain multiple series," Proceedings of the Cambridge Philosophical Society, vol. 19, pp. 86-95, 1917.

[3] F. Móricz, "Extensions of the spaces $c$ and $c_{0}$ from single to double sequences," Acta Mathematica Hungarica, vol. 57, no. 1-2, pp. 129-136, 1991.

[4] F. Móricz and B. E. Rhoades, "Almost convergence of double sequences and strong regularity of summability matrices," Mathematical Proceedings of the Cambridge Philosophical Society, vol. 104, no. 2, pp. 283-294, 1988.

[5] M. Basarir and O. Sonalcan, "On some double sequence spaces," Indian Academy of Mathematics. Journal, vol. 21, no. 2, pp. 193200, 1999.

[6] M. Mursaleen and S. A. Mohiuddine, "Double $\sigma$-multiplicative matrices," Journal of Mathematical Analysis and Applications, vol. 327, no. 2, pp. 991-996, 2007.

[7] M. Mursaleen and S. A. Mohiuddine, "Regularly $\sigma$-conservative and $\sigma$-coercive four dimensional matrices," Computers \& Mathematics with Applications, vol. 56, no. 6, pp. 1580-1586, 2008.

[8] M. Mursaleen and S. A. Mohiuddine, Convergence Methods for Double Sequences and Applications, Springer, 2014.

[9] B. Altay and F. Başar, "Some new spaces of double sequences," Journal of Mathematical Analysis and Applications, vol. 309, no. 1, pp. 70-90, 2005.

[10] F. Başar and Y. Sever, "The space $L_{p}$ of double sequences," Mathematical Journal of Okayama University, vol. 51, pp. 149157, 2009.
[11] A. Pringsheim, "Zur Theorie der zweifach unendlichen Zahlenfolgen," Mathematische Annalen, vol. 53, no. 3, pp. 289-321, 1900.

[12] J. Lindenstrauss and L. Tzafriri, "On Orlicz sequence spaces," Israel Journal of Mathematics, vol. 10, pp. 379-390, 1971.

[13] L. Maligranda, Orlicz Spaces and Interpolation, vol. 5 of Seminars in Mathematics, Polish Academy of Science, 1989.

[14] A. Wilansky, Summability through Functional Analysis, vol. 85, North-Holland Mathematics Studies, 1984.

[15] C. Cakan, B. Altay, and M. Mursaleen, "The $\sigma$-convergence and $\sigma$-core of double sequences," Applied Mathematics Letters, vol. 19, pp. 1122-1128, 2006.

[16] M. Mursaleen and S. A. Mohiuddine, "On $\sigma$-conservative and boundedly $\sigma$-conservative four dimensional matrices," Computers \& Mathematics with Applications, vol. 59, pp. 880-885, 2010.

[17] J. Musielak, Orlicz Spaces and Modular Spaces, vol. 1034 of Lecture Notes in Mathematics, Springer, Berlin, Germany, 1983.

[18] S. D. Parashar and B. Choudhary, "Sequence spaces defined by Orlicz functions," Indian Journal of Pure and Applied Mathematics, vol. 25, no. 14, pp. 419-428, 1994.

[19] K. Raj, A. K. Sharma, and S. K. Sharma, "A sequence space defined by Musielak-Orlicz function," International Journal of Pure and Applied Mathematics, vol. 67, no. 4, pp. 475-484, 2011.

[20] K. Raj, S. K. Sharma, and A. K. Sharma, "Difference sequence spaces in $n$-normed spaces defined by Musielak-Orlicz function," The Armenian Journal of Mathematics, vol. 3, no. 3, pp. 127-141, 2010.

[21] K. Raj and S. K. Sharma, "Some sequence spaces in 2-normed spaces defined by Musielak-Orlicz function," Acta Universitatis Sapientiae Mathematica, vol. 3, no. 1, pp. 97-109, 2011.

[22] H. Kizmaz, "On certain sequence spaces," Canadian Mathematical Bulletin, vol. 24, no. 2, pp. 169-176, 1981.

[23] M. Et and R. Çolak, "On some generalized difference sequence spaces," Soochow Journal of Mathematics, vol. 21, no. 4, pp. 377386, 1995. 


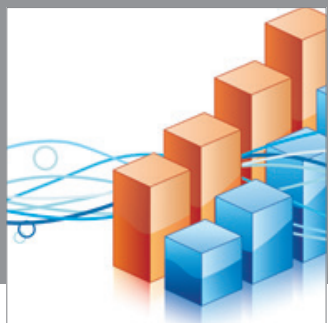

Advances in

Operations Research

mansans

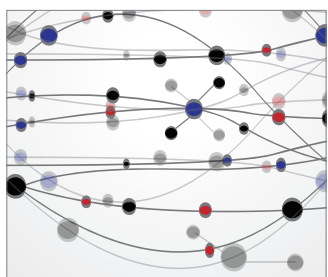

The Scientific World Journal
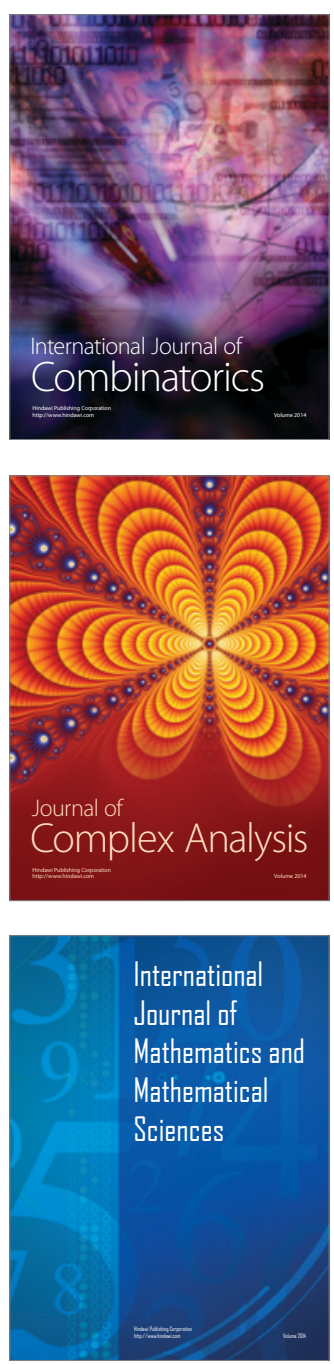
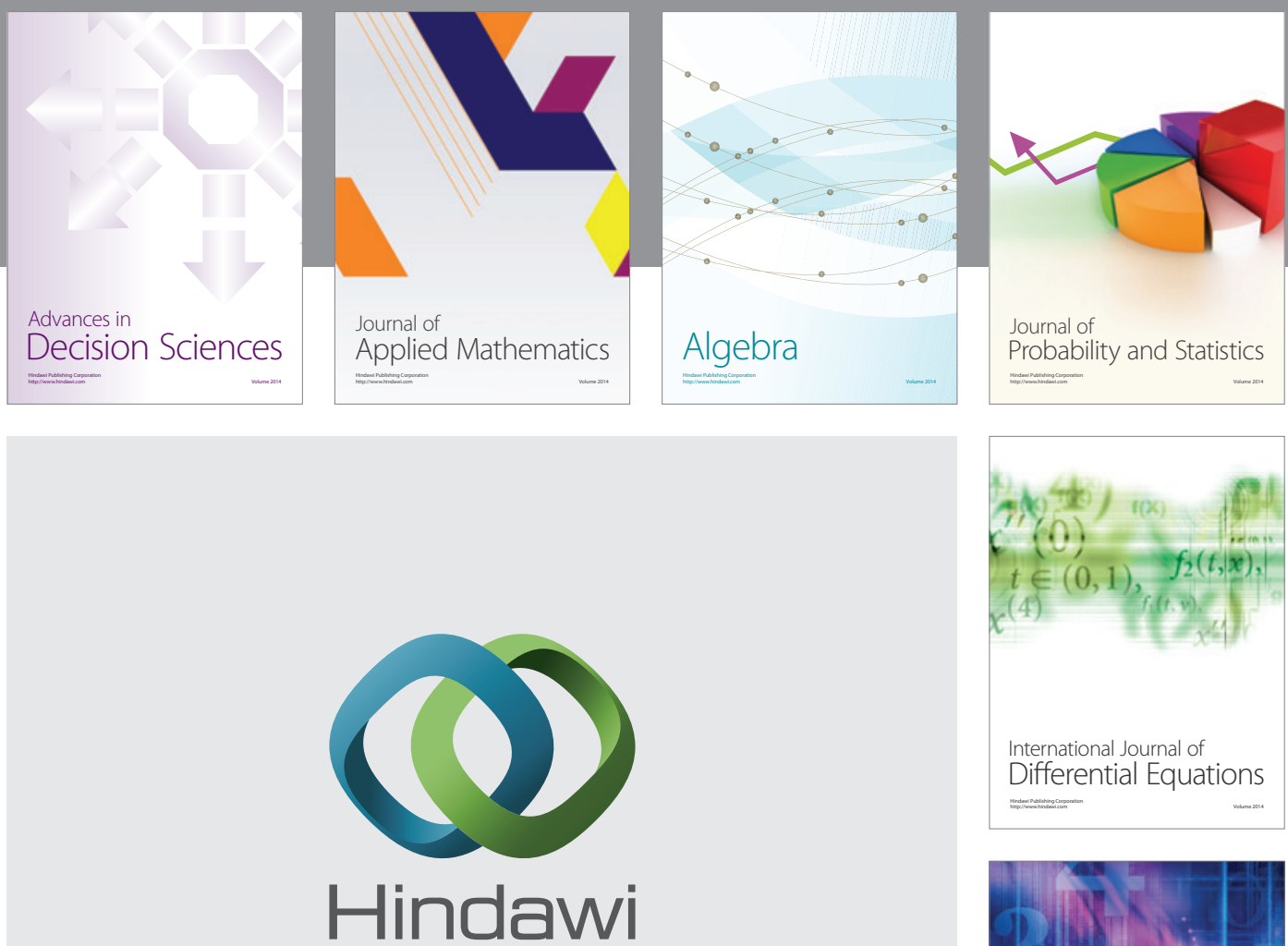

Submit your manuscripts at http://www.hindawi.com
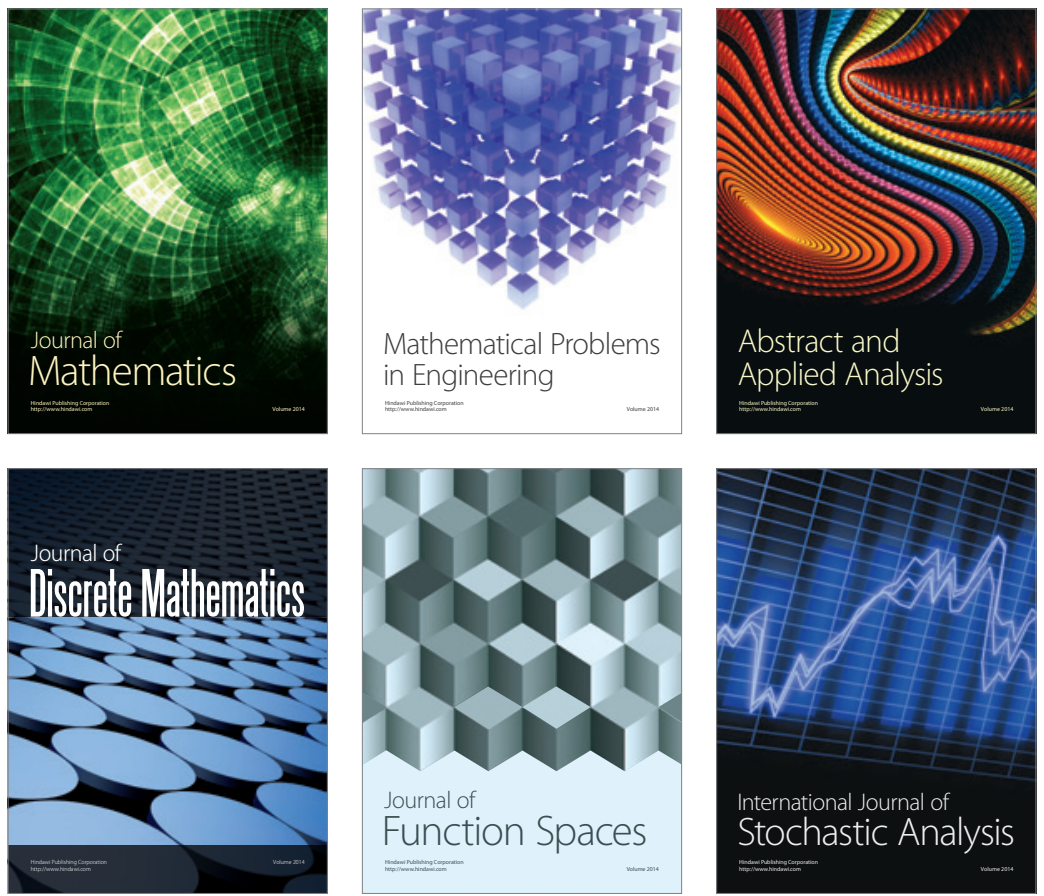

Journal of

Function Spaces

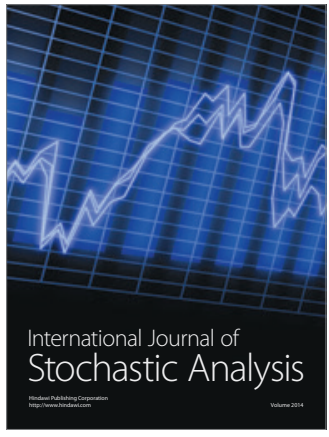

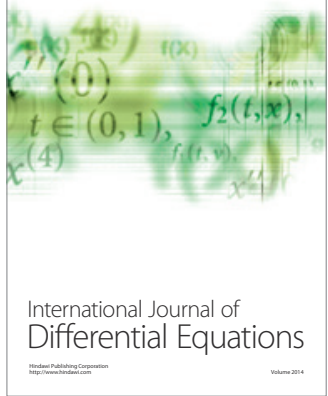
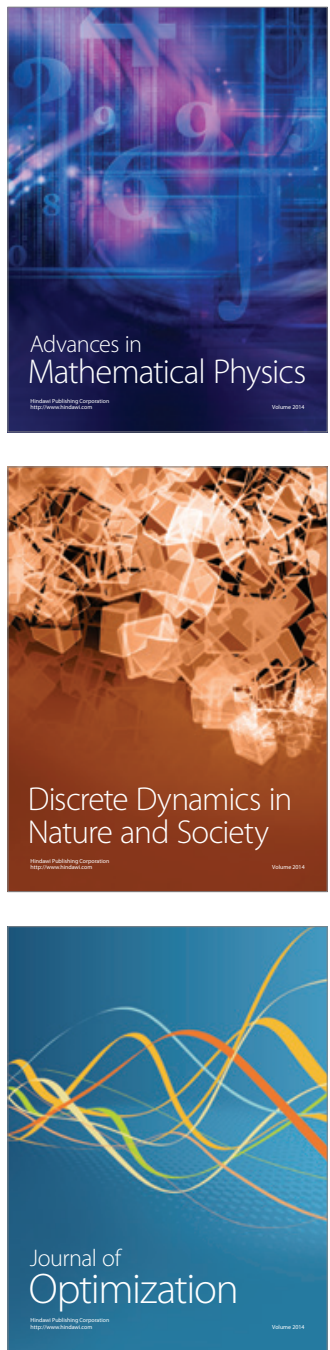